\title{
Gastritis Stages in Monozygotic and Dizygotic Dyspeptic Twins
}

\author{
Mindaugas Urba, ${ }^{1}$ Jurgita Skieceviciene, ${ }^{1}$ Dainius Janciauskas, ${ }^{2}$ Laimas Jonaitis, ${ }^{1}$ \\ Limas Kupcinskas, ${ }^{1}$ Matteo Fassan, ${ }^{3}$ Massimo Rugge, ${ }^{3,4}$ and Juozas Kupcinskas $\mathbb{D}^{1}$ \\ ${ }^{1}$ Department of Gastroenterology, Institute for Digestive Research, Lithuanian University of Health Sciences, \\ Kaunas LT-50161, Lithuania \\ ${ }^{2}$ Department of Pathological Anatomy, Lithuanian University of Health Sciences, Kaunas LT 50161, Lithuania \\ ${ }^{3}$ Department of Medicine (DIMED), Surgical Pathology \& Cytopathology Unit, University of Padua, Padua 35121, Italy \\ ${ }^{4}$ Veneto Cancer Registry, Regional Authority, Padova 35100, Italy \\ Correspondence should be addressed to Juozas Kupcinskas; juozas.kupcinskas@lsmuni.lt
}

Received 22 February 2020; Revised 30 May 2020; Accepted 1 June 2020; Published 2 July 2020

Academic Editor: Bruno Annibale

Copyright (c) 2020 Mindaugas Urba et al. This is an open access article distributed under the Creative Commons Attribution License, which permits unrestricted use, distribution, and reproduction in any medium, provided the original work is properly cited.

\begin{abstract}
Background. The progression of Helicobacter pylori-associated gastritis towards atrophic gastritis is modulated by host-related and environmental factors. Studies that explore the possible involvement of host-related versus environmental factors in the development of gastritis phenotype induced by $\mathrm{H}$. pylori are highly needed. Aims. Our study was aimed at investigating the phenotype of $H$. pylori-associated gastritis in two cohorts of monozygotic and dizygotic twins, using the OLGA/OLGIM gastritis staging system. Methods. Two cohorts of monozygotic (14 pairs) and dizygotic (15 pairs) dyspeptic twins prospectively underwent endoscopy with biopsy sampling based on Sydney protocol. H. pylori status and OLGA/OLGIM stages were assessed and compared. Results. The mean age of monozygotic and dizygotic twins was 40.4 and 38.6 years, respectively $(p=0.623)$. The overall prevalence of $H$. pylori infection was $51.7 \%$. Among the 14 monozygotic twin pairs, five pairs were $H$. pylori-positive, four were $H$. pylori-negative, and five were $H$. pylori-discordant. Among the 15 dizygotic twin pairs, five pairs were $H$. pylori-positive, five were $H$. pylori-negative, and five were $H$. pylori-discordant. Concordance for antrum atrophy in monozygotic twins was $78.6 \%$ (11/14 pairs) and in dizygotic twins $73.3 \%$ (11/15 pairs) $(p=0.742)$. Concordance for corpus atrophy in monozygotic versus dizygotic twins was $92.9 \%$ (13/14 pairs) and $86.7 \%(13 / 15$ pairs), respectively $(p=0.584)$. Concordance for antrum intestinal metaplasia (IM) in monozygotic twins was $85.7 \%$ (12/14 pairs) and in dizygotic $73.3 \%$ $(11 / 15$ pairs) $(p=0.411)$. Concordance for corpus IM in monozygotic twins was $85.7 \%(12 / 14$ pairs $)$ and in dizygotic $86.7 \%$ (13/15 pairs) $(p=0.941)$. Among monozygotic and dizygotic subjects, the stage of gastritis was concordant in both $H$. pyloripositive and $H$. pylori-negative subjects. Conclusions. In conclusion, histological gastric mucosa alterations in monozygotic and dizygotic twins showed high rates of concordance. Furthermore, OLGA/OLGIM gastritis stages were not modulated by the zygosity of the twins.
\end{abstract}

\section{Introduction}

Gastric carcinogenesis is a multistep process, including a stepwise sequence of phenotypic modifications of the native gastric tissue from healthy gastric mucosa towards atrophic gastritis (AG), intestinal metaplasia (IM), and gastric cancer (GC) [1]. Within this spectrum of lesions, gastric mucosa atrophy is considered the elective "field of cancerization" prone to GC development [1]. Helicobacter pylori (H. pylori) is by far the most common etiological agent of gastric atro- phy, and, all over the world, the prevalence of the H. pylori infection is consistently linked with both, AG and GC [1-3].

In some peculiar epidemiological contexts, however, a high prevalence of bacterial infection is associated with a low prevalence of gastric precancerous/cancer lesions, or equivalent rates of bacterial infection are associated with significantly different risk of gastric malignancy $[4,5]$. These unexpected findings would support the hypothesis that etiological factors, other than $H$. pylori, may be involved in the modulation of the oncogenetic "cascade." Among these 
factors, both host-related (genetic variations, noncoding RNAs, methylation, etc) and/or other environmental factors have been considered [6-14].

To explore the potential host-related factors in the pathogenesis of gastric atrophy, this study compares two populations of dyspeptic monozygotic and dizygotic Lithuanian twins. This is the first study in the field that comprehensively evaluates gastric mucosa alterations in twins.

\section{Materials and Methods}

2.1. Study Population. Two cohorts of twins were selected from the Twin Registry Center at Lithuanian University of Health Sciences (years 2016-2018). The twins were interviewed by phone calls, and those that reported clinical symptoms of dyspepsia were referred for upper gastrointestinal endoscopy. Zygosity of twins (mono- versus dizygosity) was confirmed by automated analysis of DNA microsatellite markers. The study included 29 twin pairs (58 subjects) older than 18 years: 14 pairs of monozygotic and 15 pairs (10 same-sex pairs and 5 mixed-sex pairs) of dizygotic twins. None of the considered patients had previously undergone anti-H. pylori treatment. All study participants did not use PPIs or antibiotics at least one month prior to inclusion.

\subsection{Information on Approval of the Local Ethical Committee} and Informed Consent. The study protocol was approved by the Ethical Committee of Lithuanian University of Health Sciences (BE-2-10). Informed consent was given for all patients before endoscopy.

2.3. Endoscopy and Protocol of Gastric Biopsy Sampling. All the endoscopic procedures were performed by the same trained gastroenterologist at the Department of Gastroenterology of Hospital of Lithuanian University of Health Sciences Kaunas Clinics in order to meet requirements for OLGA and OLGIM histological scoring [14-16]. In all patients, the biopsy protocol included 5 biopsy samples, according to the Sydney recommendations [17]. Biopsy specimens were fixed in formalin ( $10 \%$ solution), embedded in paraffin, and stained with hematoxylin and eosin and Giemsa stain for H. pylori. Gastric biopsy specimens were histologically assessed (Sydney score) by a trained pathologist, blinded to any clinical information. In all cases, both the OLGA and OLGIM staging systems were applied, according to the defined criteria $[1,18,19]$.

2.4. Statistical Analysis. Statistical analysis was performed using IBM SPSS software ( $\mathrm{v} 22.0$ ). Age is presented as mean and median values, while age differences between the groups were evaluated comparing mean values using a $T$-test. The Pearson Chi-squared test to compare sample proportion was applied. The exact Pearson Chi-squared test was used to assess concordance of OLGA and OLGIM stages between monozygotic and dizygotic twins. $p<0.05$ was considered significant.

\section{Results}

3.1. Characteristics of Subjects. Demographics and major clinical findings of twins included within the study are shown in Table 1. The mean and median age (years) of monozygotic and dizygotic twins was 40.3 (39.5) and 38.6 (36), respectively $(p=0.623)$.

3.2. H. pylori Status in Twins. The overall prevalence of $H$. pylori infection was $51.7 \%$. Concordance rates for $H$. pylori infection prevalence among monozygotic and dizygotic twins was $35.7 \%$ and $33.3 \%$, respectively $(p=0.891)$.

Among monozygotic twins, the $H$. pylori status ( $H p$ status) was as the following: five pairs were $H p$-positive, four pairs were $H p$-negative, and five pairs were $H p$-discordant. Among dizygotic twins, five pairs were $H p$-positive and five pairs were $H p$-negative, and in five pairs, the $H p$-status was discordant. Concordant $\mathrm{Hp}$-status (both $\mathrm{Hp}$-positive and $\mathrm{Hp}$-negative) in monozygotic and dizygotic twins was determined in $9 / 14(64.3 \%)$ and in $10 / 15(66.7 \%)$ twin pairs, respectively $(p=0.893)$ (Table 1$)$.

3.3. Histological Alterations of Gastric Mucosa in Twins. Concordance of topographical extension of atrophic lesions among monozygotic and dizygotic twins is shown in Table 2.

The distribution of monozygotic and dizygotic twins according to OLGA and OLGIM gastritis stages is shown in Table 3. The prevalence of low-risk OLGA and OLGIM stages among mono- and dizygotic twins was $100 \%$ and $93.3 \%$, respectively, whereas the prevalence of high-risk stages was $0 \%$ and $6.7 \%$, respectively. Concordance according to OLGA and OLGIM stages between monozygotic and dizygotic twins did not reach statistical significance $(p=0.097$ and $p=0.175$, respectively).

Tables 4 and 5 show the prevalence of similar or different gastritis stages (grouped in low versus high risk) in monozygotic and dizygotic twins distinguished by their $\mathrm{Hp}$-status (Hp-positive pair, $H p$-negative-pair, and $H p$-discordant pair). Low-risk stages (stages 0 -I-II) largely prevailed among both mono- and dizygotic twins (only two cases featured a high-risk stage (stage III, by OLGA and OLGIM). By applying both OLGA and OLGIM staging, no differences emerged in the distribution of twins by stage after performing a comparison of low- versus high-risk stages.

\section{Discussion}

Our study has investigated the phenotype of $H$. pylori-associated gastritis in two cohorts of monozygotic (14 pairs) and dizygotic (15 pairs) twins. The results of our study revealed high concordance rates of gastric mucosal alterations both in monozygotic and dizygotic twins. To our best knowledge, to date, there have been no previous reports, which have assessed the importance of shared genetic influences on susceptibility and phenotype of chronic $H$. pylori gastritis and premalignant gastric alterations on a welldefined twin cohort.

Epidemiological contexts, case-controls, and twins studies have addressed the issue of the possible interaction between different host-related and environmental risk factors 
TABle 1: Demographics and H. pylori status distinguishing monozygotic versus dizygotic twins.

\begin{tabular}{|c|c|c|c|c|c|c|c|}
\hline \multirow{2}{*}{$\begin{array}{l}\text { Twin pairs } \\
\text { Gender of twins within a pair }\end{array}$} & \multicolumn{3}{|c|}{ Monozygotic $n(14)$} & \multicolumn{3}{|c|}{ Dizygotic $n(15)$} & \multirow[t]{2}{*}{$p$ value } \\
\hline & $\begin{array}{l}\text { MM } \\
n(3)\end{array}$ & $\begin{array}{c}\text { FF } \\
n(11)\end{array}$ & $\begin{array}{c}\text { MF } \\
-\end{array}$ & $\begin{array}{l}\text { MM } \\
n(1)\end{array}$ & $\begin{array}{c}\text { FF } \\
n(9)\end{array}$ & $\begin{array}{c}\text { MF } \\
n(5)\end{array}$ & \\
\hline Mean (median) age & & $40.3(39.5)$ & & & $38.6(36)$ & & 0.623 \\
\hline Concordant $H p$-positive status & & 5 & & & 5 & & \\
\hline Concordant $H p$-negative status & & 4 & & & 5 & & 0.893 \\
\hline Discordant $H p$-negative status & & 5 & & & 5 & & \\
\hline
\end{tabular}

MM: both twins of male gender; FF: both twins of female gender; MF: twin pair comprised of a male and a female twin.

TABLE 2: Concordance of topographical extension of atrophy and IM in monozygotic and dizygotic twins.

\begin{tabular}{|c|c|c|c|c|c|}
\hline \multirow{2}{*}{ Twin pairs } & \multicolumn{2}{|c|}{ Monozygotic } & \multicolumn{2}{|c|}{ Dizygotic } & \multirow{2}{*}{$p$ value } \\
\hline & $n(14)$ & $\%$ & $n(15)$ & $\%$ & \\
\hline Concordance according to antrum athrophy & 11 & $78.6 \%$ & 11 & $73.3 \%$ & 0.742 \\
\hline Concordance according to corpus athrophy & 13 & $92.9 \%$ & 13 & $86.7 \%$ & 0.584 \\
\hline Concordance according to antrum IM & 12 & $85.7 \%$ & 11 & $73.3 \%$ & 0.411 \\
\hline Concordance according to corpus IM & 12 & $85.7 \%$ & 13 & $86.7 \%$ & 0.941 \\
\hline
\end{tabular}

IM: intestinal metaplasia.

TABle 3: Gastritis stage (OLGA and OLGIM systems) in monozygotic and dizygotic twins.

\begin{tabular}{|c|c|c|c|c|c|c|c|c|c|c|}
\hline \multirow{3}{*}{ Stage } & \multicolumn{4}{|c|}{ OLGA staging } & \multicolumn{6}{|c|}{ OLGIM staging } \\
\hline & \multicolumn{2}{|c|}{ Monozygotic twins } & \multicolumn{2}{|c|}{ Dizygotic twins } & \multirow{2}{*}{$p$ value } & \multicolumn{2}{|c|}{ Monozygotic twins } & \multicolumn{2}{|c|}{ Dizygotic twins } & \multirow{2}{*}{$p$ value } \\
\hline & $n(28)$ & $\%$ & $n(30)$ & $\%$ & & $n(28)$ & $\%$ & $n(30)$ & $\%$ & \\
\hline 0 & 18 & $64.3 \%$ & 24 & $80 \%$ & & 19 & $67.9 \%$ & 24 & $80 \%$ & \\
\hline I & 9 & $32.1 \%$ & 3 & $10 \%$ & & 8 & $28.6 \%$ & 3 & $10 \%$ & \\
\hline II & 1 & $3.6 \%$ & 1 & $3.3 \%$ & 0.097 & 1 & $3.6 \%$ & 1 & $3.3 \%$ & 0.175 \\
\hline III & - & - & 2 & $6.7 \%$ & & - & - & 2 & $6.7 \%$ & \\
\hline IV & - & - & - & - & & - & - & - & - & \\
\hline
\end{tabular}

OLGA: Operative Link on Gastritis Assessment; OLGIM: Operative Link on Gastritis/Intestinal-Metaplasia Assessment.

in the promotion of gastric precancerous lesions and gastric mucosal atrophy. These factors have been extensively studied among the patients with precancerous gastric lesions and included virulence factors of $H$. pylori strains, environmental factors (smoking, salted food, etc.), or genetic predisposition of the host $[20,21]$. To date, however, the exact factors that trigger malignant transformation from normal gastric mucosa towards gastric cancer are not completely understood.

A previous study conducted in Sweden involving monozygotic (36 pairs) and dizygotic (88 pairs) twins reared apart revealed that the concordance rate for $H$. pylori infection was significantly higher among monozygotic twins (82\%) than among dizygotic (66\%) twins [22]. Slightly higher concordance rates for $H$. pylori infection prevalence among monozygotic $(35.7 \%)$ versus dizygotic $(33.3 \%)$ have also been observed in the present study. The difference, however, did not reach statistical significance, probably due to a small number of twin pairs. Based on case-control studies in different geographical regions, which evaluate the prevalence of $H$. pylori infection and the development of gastric atrophy and IM, first-degree relatives of GC patients are associated with an increased risk for GC. A Marcos-Pinto et al. [21] study showed that first-degree relatives of early-onset gastric carcinoma patients have significantly higher prevalence of $H$. pylori, AG, and advanced stages of OLGA. H. pylori was present in $82 \%$ of cases and in $59 \%$ of controls $(p=0.001)$; AG was diagnosed in $70 \%$ of cases and in $32 \%$ of control individuals, respectively $(p<0.001)$, while OLGA stages III and IV were present in $10 \%$ and $9 \%$ in both groups, respectively $(p<0.001)$. The results of Rokkas et al. [23] meta-analysis showed similar results: their pooled OR of $H$. pylori infection, AG, and IM between individuals with family history of GC were 1.92-fold ( $p \leq 0.001), 2.2$-fold ( $p=0.005$ ), and 1.98-fold $(p \leq 0.001)$ higher, respectively, in comparison with controls. These results can be influenced by the exposition of shared environmental factors (alcohol, smoking, salted and smoked food, and hygiene) between family members, circulation of intrafamilial $H$. pylori strains [24-26], and genetic susceptibility [27-29].

Our study showed that there was no difference in concordance rates for chronic $H$. pylori gastritis among monozygotic twins $(64.3 \%, 9 / 14$ pairs) as compared to dizygotic twins $66.7 \%(10 / 15$ pairs $)(p=0.893)$. This could partly relate to the fact that twins share the same environment in early childhood and genetic predisposition for $H$. pylori 
TABLe 4: Gastritis OLGA-stage according to the H. pylori status: twins are distinguished according to zygosity.

\begin{tabular}{lccccc}
\hline \multirow{2}{*}{ Group of stages } & \multicolumn{2}{c}{ Twin pair } & \multicolumn{2}{c}{ Twin pair } & \multicolumn{2}{c}{ Twin pair } \\
& \multicolumn{2}{c}{ Hp-positive (10 pairs) } & \multicolumn{2}{c}{ Hp-negative (9 pairs) } & \multicolumn{2}{c}{$\begin{array}{c}H p \text {-discordant (10 pairs) } \\
\text { Monozygotic }\end{array}$} & Dizygotic & Monozygotic & Dizygotic & Monozygotic & Dizygotic \\
\hline Similar low-risk stage (0-I-II) & 5 & 4 & 4 & 5 & 4 \\
Similar high-risk stage (III-IV) & - & - & - & - & - \\
Discordant stage (0-I-II versus III-IV) & - & 1 & - & - & - \\
\hline
\end{tabular}

OLGA: Operative Link on Gastritis Assessment; Hp: H. pylori.

TABLe 5: Gastritis OLGIM-stage according to the H. pylori status distinguished by zygosity.

\begin{tabular}{|c|c|c|c|c|c|c|}
\hline \multirow[t]{2}{*}{ Group of stages } & \multicolumn{2}{|c|}{$\begin{array}{c}\text { Twin pair } \\
H p \text {-positive (10 pairs) }\end{array}$} & \multicolumn{2}{|c|}{$\begin{array}{c}\text { Twin pair } \\
H p \text {-negative ( } 9 \text { pairs) }\end{array}$} & \multicolumn{2}{|c|}{$\begin{array}{c}\text { Twin pair } \\
H p \text {-discordant (10 pairs) }\end{array}$} \\
\hline & Monozygotic & Dizygotic & Monozygotic & Dizygotic & Monozygotic & Dizygotic \\
\hline Similar low-risk stage (0-I-II) & 5 & 4 & 4 & 5 & 5 & 4 \\
\hline Similar high-risk stage (III-IV) & - & - & - & - & - & - \\
\hline Discordant stage (0-I-II versus III-IV) & - & 1 & - & - & - & 1 \\
\hline
\end{tabular}

OLGIM: Operative Link on Gastritis/Intestinal-Metaplasia Assessment; Hp: H. pylori.

acquisition might be less important. Concordance of topographical extension of atrophy and IM was higher among monozygotic twins but did not reach statistical significance. There were no statistical differences in concordance rates according to OLGA and OLGIM stages between monozygotic and dizygotic twins ( $p=0.097$ and 0.175 , respectively). It is very important to point out that 11 of 14 monozygotic twin pairs and 10 out of 15 dizygotic twins had absolute concordance for OLGA stages. Similarly, 10 out of 14 monozygotic twin pairs and 10 out of 15 dizygotic twins had absolute concordance for OLGIM stages. These findings suggest that if one twin is identified with high-risk premalignant gastric lesions, the other twin should also be assessed for these alterations.

A relatively small number of the study population represent the main limitation of the present study. The numbers of individual twins within our study group were not very large, and certain effects might have been biased. The design of the study also did not allow us to evaluate as to what extent gastric mucosal alterations in twins are related to the common shared genetic factors per se or shared environmental factors during childhood and later on in lifetime. There might also have been a certain selection bias, because our study included only twins with dyspeptic symptoms.

\section{Conclusions}

In conclusion, histological gastric mucosa alterations in monozygotic and dizygotic twins showed high rates of concordance. Furthermore, OLGA and OLGIM gastritis stages were not modulated by the zygosity of the twins.

\section{Data Availability}

The authors can make research data available on request after institutional review board approval through contacting the corresponding author by juozas.kupcinskas@lsmuni.lt

\section{Conflicts of Interest}

The authors declare that there is no conflict of interest regarding the publication of this paper.

\section{Acknowledgments}

We would like to acknowledge Justina Arstikyte for excellent work in the laboratory. The work was supported by the grant of the Research Council of Lithuania (APP-2/2016).

\section{References}

[1] R. M. Genta and M. Rugge, "Gastric precancerous lesions: heading for an international consensus," Gut, vol. 45, Supplement 1, pp. I5-I8, 1999.

[2] F. Farinati, R. Cardin, V. M. Russo, G. Busatto, M. Franco, and M. Rugge, "Helicobacter pylori CagA status, mucosal oxidative damage and gastritis phenotype: a potential pathway to cancer?," Helicobacter, vol. 8, no. 3, pp. 227-234, 2003.

[3] H. R. Ghasemi Basir, M. Ghobakhlou, P. Akbari, A. Dehghan, and M. A. Seif Rabiei, "Correlation between the intensity of Helicobacter pylori colonization and severity of gastritis," Gastroenterology Research and Practice, vol. 2017, 5 pages, 2017.

[4] V. V. Tsukanov, N. N. Butorin, A. S. Maady et al., "Helicobacter pylori infection, intestinal metaplasia, and gastric cancer risk in Eastern Siberia," Helicobacter, vol. 16, no. 2, pp. 107112, 2011.

[5] V. V. Tsukanov, E. V. Kasparov, J. L. Tonkikh et al., "Peptic ulcer disease and Helicobacter pylori infection in different Siberian ethnicities," Helicobacter, vol. 22, no. 1, 2017.

[6] F. Petersson, K. Borch, and L. E. Franzén, "Prevalence of subtypes of intestinal metaplasia in the general population and in patients with autoimmune chronic atrophic gastritis," Scandinavian Journal of Gastroenterology, vol. 37, no. 3, pp. 262-266, 2009.

[7] S. K. M. Heinrichs, T. Hess, J. Becker et al., "Evidence for PTGER4, PSCA, and MBOAT7 as risk genes for gastric cancer 
on the genome and transcriptome level," Cancer Medicine, vol. 7, no. 10, pp. 5057-5065, 2018.

[8] L. Kupcinskas, T. Wex, J. Kupcinskas et al., "Interleukin-1B and interleukin-1 receptor antagonist gene polymorphisms are not associated with premalignant gastric conditions: a combined haplotype analysis," European Journal of Gastroenterology and Hepatology, vol. 22, no. 10, pp. 1189-1195, 2010.

[9] V. Petkevicius, V. Salteniene, S. Juzenas et al., "Polymorphisms of microRNA target genesIL12B,INSR,CCND1andIL10in gastric cancer," World Journal of Gastroenterology, vol. 23, no. 19, pp. 3480-3487, 2017.

[10] A. Link and J. Kupcinskas, "MicroRNAs as non-invasive diagnostic biomarkers for gastric cancer: current insights and future perspectives," World Journal of Gastroenterology, vol. 24, no. 30, pp. 3313-3329, 2018.

[11] D. B. Polk and R. M. Peek, “_Helicobacter pylori_: gastric cancer and beyond," Nature Reviews. Cancer, vol. 10, no. 6, pp. 403-414, 2010.

[12] M. Amieva and R. M. Peek, "Pathobiology of _Helicobacter pylori_-induced gastric cancer," Gastroenterology, vol. 150, no. 1, pp. 64-78, 2016.

[13] K. Abdeljawad, A. Wehbeh, and E. Qayed, "Low prevalence of clinically significant endoscopic findings in outpatients with dyspepsia," Gastroenterology Research and Practice, vol. 2017, 7 pages, 2017.

[14] M. Rugge, K. Sugano, C. Scarpignato, D. Sacchi, W. J. Oblitas, and A. G. Naccarato, "Gastric cancer prevention targeted on risk assessment: gastritis OLGA staging," Helicobacter, vol. 24, no. 2, article e12571, 2019.

[15] M. Rugge, R. M. Genta, M. Fassan et al., "OLGA gastritis staging for the prediction of gastric cancer risk: a long-term followup study of 7436 patients," American Journal of Gastroenterology, vol. 113, no. 11, pp. 1621-1628, 2018.

[16] M. Rugge, A. Meggio, C. Pravadelli et al., "Gastritis staging in the endoscopic follow-up for the secondary prevention of gastric cancer: a 5-year prospective study of 1755 patients," Gut, vol. 68, no. 1, pp. 11-17, 2018.

[17] M. F. Dixon, R. M. Genta, J. H. Yardley, and P. Correa, "Classification and grading of gastritis," American Journal of Surgical Pathology, vol. 20, no. 10, pp. 1161-1181, 1996.

[18] M. Rugge, R. M. Genta, D. Y. Graham et al., "Chronicles of a cancer foretold: 35 years of gastric cancer risk assessment," Gut, vol. 65, no. 5, pp. 721-725, 2016.

[19] M. Rugge, G. Pennelli, E. Pilozzi et al., "Gastritis: the histology report," Digestive and Liver Disease, vol. 43, SUPPLEMENT 4, pp. S373-S384, 2011.

[20] W. J. den Hollander, I. L. Holster, C. M. den Hoed et al., "Surveillance of premalignant gastric lesions: a multicentre prospective cohort study from low incidence regions," Gut, vol. 68, no. 4, pp. 585-593, 2019.

[21] R. Marcos-Pinto, F. Carneiro, M. Dinis-Ribeiro et al., "Firstdegree relatives of patients with early-onset gastric carcinoma show even at young ages a high prevalence of advanced OLGA/OLGIM stages and dysplasia," Alimentary Pharmacology and Therapeutics, vol. 35, no. 12, pp. 1451-1459, 2012.

[22] H. M. Malaty, D. Y. Graham, I. Isaksson, L. Engstrand, and N. L. Pedersen, "Are genetic influences on peptic ulcer dependent or independent of genetic influences for Helicobacter pylori infection?", Archives of Internal Medicine, vol. 160, no. 1, pp. 105-109, 2000.
[23] T. Rokkas, P. Sechopoulos, D. Pistiolas, G. Margantinis, and G. Koukoulis, "Helicobacter pylori infection and gastric histology in first-degree relatives of gastric cancer patients: a metaanalysis," European Journal of Gastroenterology and Hepatology, vol. 22, no. 9, pp. 1128-1133, 2010.

[24] J. Raymond, J.-M. Thiberge, C. Chevalier et al., "Genetic and transmission analysis of Helicobacter pylori strains within a family," Emerging Infectious Diseases, vol. 10, no. 10, pp. 1816-1821, 2004.

[25] M. Kivi, Y. Tindberg, M. Sorberg et al., "Concordance of Helicobacter pylori strains within families," Journal of Clinical Microbiology, vol. 41, no. 12, pp. 5604-5608, 2003.

[26] M. Konno, N. Fujii, S.-i. Yokota et al., "Five-year follow-up study of mother-to-child transmission of Helicobacter pylori infection detected by a random amplified polymorphic DNA fingerprinting method," Journal of Clinical Microbiology, vol. 43, no. 5, pp. 2246-2250, 2005.

[27] F. Li, C. Huang, Q. Li, and X. Wu, "Construction and comprehensive analysis for dysregulated long non-coding RNA (lncRNA)-associated competing endogenous RNA (ceRNA) network in gastric cancer," Medical Science Monitor, vol. 24, pp. 37-49, 2018.

[28] X. Cui, Q. Huang, X. Li et al., "Relationship between interleukin-10 gene C-819T polymorphism and gastric cancer risk: insights from a meta-analysis," Medical Science Monitor, vol. 22, pp. 2839-2845, 2016.

[29] B. Chen, M. X. Luo, X. Zhou, Y. Lv, and G. Q. Su, "Correlation between interleukin-1 $\beta-511 \mathrm{C} / \mathrm{T}$ polymorphism and gastric cancer in Chinese populations: a meta-analysis," Medical Science Monitor, vol. 22, pp. 1742-1750, 2016. 